\title{
Maison des Étudiants de Nantes : le dispositif «Univers-cité »
}

http://www.univers-cite.org/

Laurence Marie

\section{OpenEdition}

\section{Journals}

Édition électronique

URL : http://journals.openedition.org/labyrinthe/1196

DOI : $10.4000 /$ labyrinthe. 1196

ISSN : 1950-6031

\section{Éditeur}

Hermann

\section{Édition imprimée}

Date de publication : 1 octobre 2001

Pagination : 113-114

\section{Référence électronique}

Laurence Marie, «Maison des Étudiants de Nantes : le dispositif « Univers-cité » », Labyrinthe [En ligne], 10 | 2001, mis en ligne le 04 avril 2006, consulté le 02 mai 2019. URL : http://journals.openedition.org/ labyrinthe/1196; DOI : 10.4000/labyrinthe.1196

Ce document a été généré automatiquement le 2 mai 2019.

Propriété intellectuelle 


\title{
Maison des Étudiants de Nantes : le dispositif «Univers-cité »
}

\author{
http://www.univers-cite.org/
}

\section{Laurence Marie}

1 Les mémoires de maîtrise ou de DEA condamnés à rester au placard ? Pas lorsqu'on étudie à l'université de Nantes. Depuis la rentrée 2001, chaque étudiant nantais peut désormais plancher sur des sujets de recherche proposés par les collectivités locales et les entreprises de la région, puis validés par un enseignant. Philipe Quéré, président de la Maison des Étudiants de Nantes précise l'objectif d'« Univers-Cité » : « nous voulons offrir à chaque étudiant la possibilité de faire de ses travaux de recherche des documents utiles à la société et de recevoir en retour une meilleure reconnaissance de la valeur de ces travaux.» Plus de trois ans ont été nécessaires pour mettre en place le dispositif: regrouper les partenaires - la Chambre de Commerce et d'Industrie de Nantes, le Conseil Régional des Pays de la Loire, le Conseil de Développement de la Communauté Urbaine -, tester les idées auprès des professionnels, et signer une convention avec l'université. Commentaire de Philippe Quéré : "L'idée de départ était simple, mais il nous a fallu beaucoup de persévérance car en demandant l'accès aux mémoires des étudiants, nous touchions aux prérogatives de l'université. »

Certains sujets, très précis, ne seront traités que par un étudiant : «Le recensement des équipements sportifs de l'agglomération nantaise, cartographie des pratiques sportives, besoins exprimés ", "Comment la mise en œuvre d'une gestion du temps des habitants peut générer une nouvelle approche des problématiques d'aménagement », «L'impact de la route sur la faune et la flore", "L'évolution historique du littoral de la LoireAtlantique ». Mais la plupart des sujets, plus thématiques, sont proposés à plusieurs étudiants, chacun s'y attelant selon ses intérêts personnels, en multipliant les angles d'approche. En témoignent ces études demandées par le Conseil Général des Pays de la Loire : «L'eau », «Le développement local», «Les services publics locaux», ou encore «La gestion des territoires».

D'autres outils sont en cours d'élaboration. Un annuaire des propositions de sujets et une revue électronique diffusée par e-mail informeront les abonnés des moyens d'interaction 
entre l'université et les acteurs de la société. Sans compter que l'équipe d'« Univers-Cité » étudie également la possibilité pour les étudiants ayant déjà validé leurs travaux de présenter directement leur recherche aux entreprises intéressées. Avec là encore un double bénéfice : pas de délai d'attente pour les entreprises et une rémunération possible pour l'étudiant.

4 Une vingtaine d'entreprises sont à présent abonnées à «Univers-Cité». Et les institutionnels ont salué l'importance d'un dispositif qui permet de « faciliter l'ouverture sur les métiers et la capacité des étudiants à entrer dans la vie professionnelle » (Yann Tanguy, Président de l'université de Nantes) et de « faire de la Région des Pays de la Loire un des territoires les mieux équipés en matière de nouvelles technologies " (François Fillion, Président du Conseil Régional des Pays de la Loire). Reste à souhaiter qu'« Univers-Cité » fasse des émules. La Maison des Étudiants de Nantes est en contact avec trois autres universités de la région... 